\title{
PET/MR in neuro-oncology: is it ready for prime-time?
}

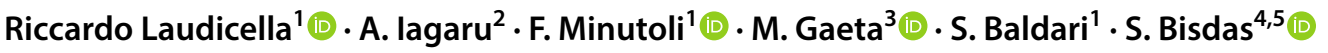

Received: 30 March 2020 / Accepted: 21 June 2020 / Published online: 26 June 2020

(c) Italian Association of Nuclear Medicine and Molecular Imaging 2020

Since 1982, when Di Chiro et al. published their first cancer study using [18F] fluorodeoxyglucose (FDG) positron emission tomography (PET) to show that FDG uptake was higher in high-grade gliomas (HGG) than in low-grade gliomas (LGG), several steps have been taken in the metabolic imaging of brain tumours, much advanced by the clinical PET/ computed tomography (CT). In brain tumours, magnetic resonance (MR) provides substantial diagnostic advantages over CT. Over the past four decades, we have witnessed a shift in neuro-oncologic imaging demand from merely structural and anatomical information toward additional information about tumour physiology. Although MR imaging may provide useful structural and anatomical information, the gadolinium enhancement is unspecific for the tumour grading and advanced specialized MR techniques investigating tumoral metabolic features, such as proton/phosphor/sodium spectroscopy, and iron-oxide particles contrast agents, do not offer either high-spatial-resolution or have considerable operator/scanner variability and are not widely employed. Beyond tumour staging, conventional MR suffers from compromised specificity in tumour surveillance in the presence of treatment-related changes. Therefore, the additional insights provided by molecular imaging in the clinical management and pathophysiology of brain tumours is essential.

Riccardo Laudicella

riclaudi@hotmail.it

$1 \quad$ Nuclear Medicine Unit, Department of Biomedical and Dental Sciences and Morpho-Functional Imaging, University of Messina, Via Consolare Valeria n.1, Messina, Italy

2 Division of Nuclear Medicine and Molecular Imaging, Stanford University School of Medicine, Stanford, USA

3 Section of Radiological Sciences, Department of Biomedical Sciences and Morphological and Functional Imaging, University of Messina, Messina, Italy

4 Department of Neuroradiology, University College London Hospitals NHS Trust, London, UK

5 Department of Brain Repair and Rehabilitation, University College London, London, UK
Recently, diagnostic shortcomings have been successfully addressed by a significant increase in the use of PET in the neuro-oncological field, especially for amino-acidic tracers in the evaluation of the main clinical step. The obvious synergies between MR and PET in brain tumours have hence provided the rationale to develop the simultaneous PET/MR, also to improve patient compliance and promote workflow reducing the logistical drawbacks due to sequential PET/ CT-MR acquisitions. Apart from the synchronous anatomical, microstructural (i.e., diffusion tensor imaging), functional (i.e., perfusion, diffusion, resting-state and task-based functional MR), and metabolic information from hybrid PET/MR, the technique is radiation-free, hence suitable for pediatric patients and frequent follow-up. Furthermore, it enables real-time motion correction, PET partial-volume averaging correction, pharmacokinetic modelling of the PET and MR tracers by simultaneous dynamic acquisitions. Also, due to the increased sensitivity of the used avalanche photodiodes, PET/MR can potentially allow to decrease the administered radioactivity maintaining though excellent diagnostic quality [1]. The key players for state-of-the-art PET in neuro-oncology are the aminoacidic tracers, which are largely independent from the blood-brain barrier integrity and hence outperform gadolinium by staging both enhancing and non-enhancing brain tumours. In fact, due to its high physiological uptake in normal brain grey-matter and in inflammatory lesions, FDG plays a limited role in neuro-oncology. Therefore, through a high tumour/nontumour contrast, aminoacidic tracers boosted the use of PET in neuro-oncology enabling precise tumour delineation, biopsy-planning and differentiation between recurrence and post-therapeutic changes. Aminoacidic tracers also improved the accuracy as in LGG and in HGG, with greater specificity than standard MR for the evaluation of progressive tumour and prognosis for specific therapy (i.e., anti-angiogenetic drugs). Also, and differently from FDG, aminoacidic tracers are characterized by an absent/low-uptake in acute ischaemic stroke/reperfusion, demyelinating processes, CNS infectious processes. Despite the obvious advantages, the dissemination of the PET/MR technique is rather limited and we 
consider that clear, broadly and solid clinical indications can promote its use, such as (1) primary tumour staging in case of indeterminate MR findings (i.e., non-enhancing, non "glioblastoma-typical" tumours, Fig. 1); (2) metabolic mapping in extensive tumour infiltration to identify the "hotspot" for brain biopsy guidance; (3) pre-treatment tumour surveillance to choose the optimal intervention time-point; (4) tumour surveillance to differentiate between recurrence and treatment-related changes (including pseudo-progression) when clinically suspected and structural MR is inconclusive. PET/MR in the aforementioned scenarios offers added diagnostic value and presumably reaches $100 \%$ accuracy if it is combined with advanced MR techniques (mostly dynamic susceptibility contrast MR perfusion imagingDSC - and spectroscopy). An obvious reason for this superior performance is also the detailed metabolic and functional mapping that fully captures the tumour heterogeneity, increasing the chance to identify even tiny nests of disease and therefore lead to an accurate diagnosis. However, this is a rather empirical observation, and well-designed studies are required to show the added value of each modality under the aforementioned clinical scenarios, to gauge the impact on the patient management and to create the body of evidence for the regulatory and commissioning authorities. Previous comparative stand-alone MR, PET vs. combined PET/MR studies have been methodologically flawed by under-representing the potential of advanced MR, or by conducting suboptimal PET measurements (i.e., lack of dynamic studies). Expanding the "first-line" PET/MR applications, thoughts have been made to use it for radiotherapy treatment planning and dose-painting by delineating the most active residual tumour parts, to differentiate between metastatic and primary brain tumours, and for intra-treatment monitoring during concurrent chemoradiation. The latter application appears though quite high-risk since confounding acute therapy effects probably compromise the diagnostic performance of both modalities. A key concept for integrating PET/MR into the clinical routine is, besides the economic costs, to address the time constraints: beyond the research realm, where PET/MR protocols can be long and comprehensive, the clinical practice dictates approximately $30 \mathrm{~min}$ PET/MR acquisitions. As PET is acquired synchronously (preferably in LIST mode for dynamic measurements), the MR is the main driver here and careful selection of timeefficient MR sequences is needed, also considering that gadolinium injection (or off-label iron-oxide particles) for post-contrast imaging is mandatory. Recent advances in MR, including artificial intelligence (AI)-based image reconstruction, allow to perform key functional and metabolic sequences in $50 \%$ less time than before. In the era of AI, PET/MR has the potential to re-invent diagnostics. We have a brilliant opportunity to generate evidence for the role of PET/MR "hybrid radiomics" to improve diagnostic accuracy and non-invasive molecular phenotyping (i.e., isocitrate dehydrogenase mutation), serving as a decision-tool for patient management $[2,3]$. This is particularly important in the post-treatment setting and immunotherapy regimens, where invasive biopsies are generally avoided, and neurooncologists would be tempted to understand the molecular tumour. Furthermore, an interesting approach is to combine the PET/MR radiomics with other-omics, such as genomics, metabolomics, and proteomics, to elucidate the tumourigenesis and create new non-invasive genotyping biomarkers. Today, in addition to methionine (limited by the need of an-site cyclotron), new PET tracers are part of the molecular imaging portfolio in brain tumours, such as

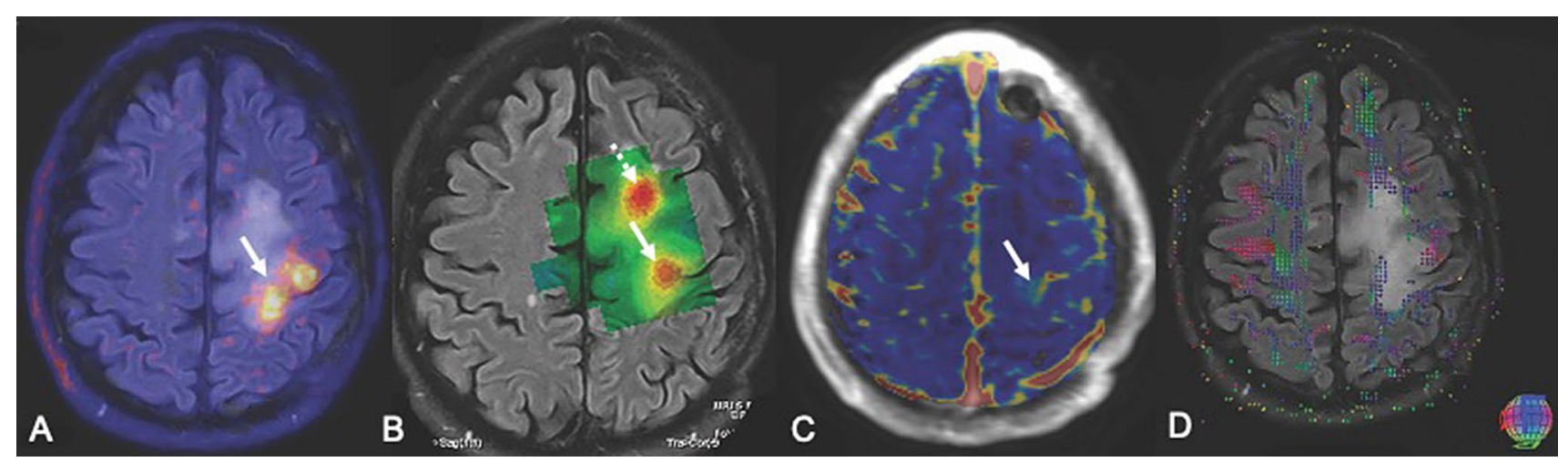

Fig. 1 Hybrid Methionine PET/MR imaging in a 45-year-old patient with a non-enhancing glioma on the left frontal gyrus. The fused PET-FLAIR-T2w image (a) shows avid radiolabeled amino-acid tracer uptake on the posterior part of the tumour in the precentral gyrus (arrow), whereas the proton MR spectroscopy Choline map (b) demonstrates a second focus of increased cell proliferation on the anterior part of the lesion (dashed arrow). The perfusion MR (intra- vascular blood volume map) (c) shows in concordance with the PET a "hot-spot" with increased vascularity on the precentral gyrus (arrow). Due to the eloquent character of the metabolically active lesion site (motor strip), simultaneous diffusion tensor imaging (DTI) was acquired to determine the location of the pyramidal motor track and help to guide the neurosurgical biopsy, thus avoiding motor function impairment 
Fluoro-ethyl-tyrosine (FET), whose time activity curves estimation represents a valuable tool to further differentiate between HGG and LGG, characterizing doubtful lesions and resulting useful in radiation therapy planning [4]. An innovative approach, akin to the use of two MR contrast agents for comprehensive MR, is exploited by Fluorothymidine (FLT) in staging HGG with inconclusive imaging features and the response to therapy assessment. Hypoxia-related tracers (i.e., Fluoromisonidazole-FMISO) can map and measure tumour hypoxia, enhancing the possibility of delivering hypoxia-modifying treatment strategies, potentially improving patient prognosis. Though not established in the clinical routine, FLT and FMISO have great potential to provide biomarkers in clinical trials. Also, prostate-imaging tracers (i.e., Choline, Fluciclovine, and prostate-specific membrane antigen-PSMA) are considered in neuro-oncology due to their lack of uptake in healthy brain tissue [5], whilst in meningiomas, the utmost tracers are represented by $68 \mathrm{Ga}$-DOTA-peptides. Other early promising results are described for Immuno-PET by using antibodies as targeting probes (i.e., ${ }^{89} \mathrm{Zr}$-labeled monoclonal antibody), able to noninvasively assess the in vivo expression levels of tumourrelated antigens $[6,7]$. The use of PET/MR as a prime imaging modality in the research scenario seems advantageous, and its implementation will potentially boost drug discovery, enabling rapid decision-making in clinical trials. Namely, we consider that the combination of the above-mentioned radiopharmaceuticals with MR may further improve the accuracy in several aspects of brain tumour management, establishing an eminent role for PET/MR in providing tailored treatment or tracking the spatial progression and distribution of the immuno-response. In conclusion, the technique has technically matured since its introduction in 2009, and the preliminary evidence shows that it is ready for prime-time clinical applications. In neuro-oncology, PET and MR are not mutually redundant nor competitive: both are equivalent "key-players" and pillars of the patient management. The combination of MR and PET in a single hybrid technique increases the diagnostic performance of each "stand-alone" modality, improving the patients' comfort, and the PET/MR suite workflow [4]. PET/MR is a "one-stopshop" imaging that paves wide and interesting pathways on research and clinical applications. The reimbursement might be the most difficult impediment to overcome. As in all novel techniques in healthcare, international consensus and level I-II evidence will qualify the use of PET/MR. The term "PET/MR imaging experts" should be inclusive for both radiologists and nuclear medicine physicians with an established training curriculum, who shall think "out-of-the-box". Our focus is to enhance our capability to improve patient management rather than underuse the method in a self-fulfilling and short-sighted manner, showing protectionism for our discipline. Training and cultivating a critical mass of specialized PET/MR experts is mandatory to establish PET/ MR as core modality in Neuro-oncology. We ought to create awareness in the public and patient groups, in oncology disciplines, and in the pharmaceutical companies about the impact of such modality on the disease course and management. PET/MR is an indispensable tool for clinical decision-making.

\section{Consent to participate}

For this type of study, formal consent is not required. All applicable international, national, and/or institutional guidelines for the care and use of animals were followed.

Acknowledgements Declared none.

Funding None.

\section{Compliance with ethical standards}

Conflict of interest All the authors declare that they have no conflict of interest related to this spotlight.

Ethical approval All procedures performed in studies involving human participants were in accordance with the ethical standards of the institutional and/or national research committee and with the 1964 Helsinki Declaration and its later amendments or comparable ethical standards.

\section{References}

1. Wehrl HF, Sauter AW, Divine MR et al (2015) Combined PET/ MR: a technology becomes mature. J Nucl Med 56:165-168

2. Lohmann P, Lerche C, Bauer E et al (2018) Predicting IDH genotype in gliomas using FET PET radiomics. Sci Rep 8:13328

3. Bisdas S, Shen H, Thust $\mathrm{S}$ et al (2018) Texture analysis- and support vector machine-assisted diffusional kurtosis imaging may allow in vivo gliomas grading and IDH-mutation status prediction: a preliminary study. Sci Rep 8:6108

4. Pyka T, Hiob D, Preibisch C et al (2018) Diagnosis of glioma recurrence using multiparametric dynamic $18 \mathrm{~F}$-fluoroethyl-tyrosine PET-MRI. Eur J Radiol 103:32-37

5. Alongi P, Vetrano IG, Fiasconaro E et al (2019) Choline-PET/ $\mathrm{CT}$ in the differential diagnosis between cystic glioblastoma and intraparenchymal hemorrhage. Curr Radiopharm 12:88-92

6. Quartuccio N, Laudicella R, Mapelli P et al (2020) Hypoxia PET imaging beyond ${ }^{18} \mathrm{~F}$-FMISO in patients with high-grade glioma: ${ }^{18} \mathrm{~F}-\mathrm{FAZA}$ and other hypoxia radiotracers. Clin Transl Imaging 8:11-20

7. Shooli H, Dadgar H, Wáng YJ et al (2019) An update on PETbased molecular imaging in neuro-oncology: challenges and implementation for a precision medicine approach in cancer care. Quant Imaging Med Surg 9:1597-1610

Publisher's Note Springer Nature remains neutral with regard to jurisdictional claims in published maps and institutional affiliations. 